\title{
Research International Journal of Cardiology and Cardiovascular Medicine
}

\section{Short Communication}

\section{Mesenteric Malperfusion in}

\section{Aortic Dissection: Diagnostic Challenge and Therapeutic \\ Options}

\author{
Dr. Jasima Nilofer ${ }^{1}$ and Dr. Mohammed Idhrees ${ }^{2 *}$ \\ ${ }^{1}$ Department of Pathology, Sree Balaji Medical College and Hospital, Chrompet, Chennai. \\ ${ }^{2}$ Cardiovascular and thoracic Surgeon, Institute of Cardiac and Aortic Disorders (ICAD), SRM Institute of \\ Medical Sciences (SIMS Hospital), Vadapalani, Chennai.
}

Received: 10 February, 2021

Accepted: 05 March, 2021

Published: 08 March, 2021

*Corresponding author: Dr. A. Mohammed Idhrees MCh, FAIS, Cardio Thoracic and Vascular Surgeon, Institute for Cardiac and Aortic Disorders, SRM Institute for Medical Sciences (SIMS Hospitals), Chennai, India. E mail: a.m.idhrees@gmail.com

Keywords: Acute aortic dissection, Mesentric malperfusion, Bowel ischemia.

Copyright: (C) 2021 Idhrees M, et al. This is an open access article distributed under the Creative Commons Attribution License, which permits unrestricted use, distribution, and reproduction in any medium, provided the original work is properly cited.

\section{Introduction}

Malperfusion in Acute Type A aortic dissection (ATAAD) has a direct cataclysmic effect on morbidity and mortality, despite the advancements in diagnosis, techniques, and technology. Malperfusion syndrome is a complication caused by branch-vessel involvement and resulting in endorgan ischemia dysfunction with an incidence ranging from $16 \%$ to $34 \%$ $[1,2]$. The complete obstruction of the vessel will present with full-blown symptoms, but it is also important to recognize incomplete or subtotal vessel occlusion which may produce intermittent symptoms of variable intensity. Mesenteric malperfusion (MMP), the most dreadful of all malperfusion, is associated with a 3-to 4-fold increase in mortality. The optimal therapeutic management of patients with malperfusion is still a topic of debate.

\section{Mechanisms of Malperfusion}

The relationship between the true lumen, false lumen and the mobile intimo-medial flap is complex. The pressure difference between both these two lumens will allow the mobile flap to bulge and cause static obliteration of branch vessel ostia. The hypercoagulable state of the false lumen provides a nidus for thrombosis leading to impaired perfusion of the end organ [3]. The mobile flap may protrude intermittently into the ostium of the branch vessel causing a dynamic obstruction.

\section{Dilemma in MMP}

MMP is extremely challenging for diagnosis and management. Since the symptoms are nonspecific, there is a delay in referral from the primary care centre to tertiary hospitals. It is intriguing to note that $20 \%$ of patients with abdominal pain do not have MMP, and $40 \%$ of patients with MMP do not have abdominal pain $[4,5]$. As compared to symptoms of other end organ failure like stroke, anuria, loss of pulses in the limbs, the symptoms of MMP are very vague. The debate of whether the patient should undergo central repair followed by mesenteric evaluation or vice versa continues. Visceral malperfusion can occur at any stage of the disease process - at the time of aortic dissection, false lumen thrombosis, during surgery or even after the repair. There are anecdotal reports of visceral ischemia which has happened even after 8 days after the aortic repair [6].
The ischemia of the gut will lead to disruption of the gut mucosal barrier releasing endotoxins. This is further insulted in the setting of ATAAD. Hepatic macrophages produce TNF (Tumour necrosis factor) causing reperfusion injury. This reperfusion is bound to happen even after central aortic repair, which cannot be controlled. These factors place this clinical condition with high mortality, with most of the patients dying within the first 24 hours even though adequate mesenteric perfusion is established [7].

\section{Management}

The objectives in management would be

(i) Decision of which pathology to be addressed first - dissection or malperfusion

(ii) Perfusion of the occluded vessel

(iii) Measures to encounter the reperfusion injury

\section{Acute Type A Aortic Dissection}

The options for patient with MMP and ATAAD include

a) Immediate central aortic repair approach: Conventionally, in any patient of ATAAD, restoring blood into the true lumen and excising the entry point in ascending aorta is the gold standard treatment. Even with the best of efforts, $25 \%$ of the patients persist to have ischemia of the branch vessels $(8,9)$.

b) Endovascular followed by central repair: With the advancement of techniques and technology in endovascular, many centers now advocate a primary endovascular management followed by central aortic repair. Recently Michigan group published their 20 years' experience, where $96 \%$ of patients either survived to discharge or underwent delayed open repair $(69.5 \%)$. The physician must differentiate between patients who have ischemic but not necrotic bowel from those with necrotic bowel. Endovascular stenting of the branch vessel to restore blood to the branch vessel has been tried in hemodynamically stable patients.

c) Simultaneous Approach: This can be approached with anyone of the below options - resection of the necrotic bowel is performed followed by central repair or a continuous perfusion of the 
Citation: Idhrees M, Nilofer J (2021) Mesenteric Malperfusion in Aortic Dissection: Diagnostic Challenge and Therapeutic Options. Rea Int J of Card and Cardio Med: 001-002. DOI: 10.37179/rijccm.000006

mesenteric artery during central repair followed by bypass to the mesenteric artery.

d) Mechanism Specific Approach: Surgeons from Hokkaido University, Japan proposed an interesting concept, where they approach these patients based on the mechanism of the malperfusion [10]. After evaluation of the dynamicity of the intimomedial flap, they will follow" Immediate central aortic repair approach", if it is an aortic-type malperfusion or a "Endovascular followed by central repair" if it is a branch-type malperfusion.

We have previously published our protocol of managing such patients in our unit [7].

\section{Acute Type B Aortic Dissection (ATBAD)}

Since the dawn of endografts, the outcomes of patients undergoing thoracic endovascular aortic repair are superior to open surgery in ATBAD [11,12]. Techniques to mitigate MMP include central aortic fenestration and/or branch artery stenting. A static obstruction of the branch vessel can be managed with an Endograft to the descending thoracic aorta.

\section{References}

1. Czerny M, Schoenhoff F, Etz C, Lars E, Nawid K, et al. (2015) The Impact of Pre-Operative Malperfusion on Outcome in Acute Type A Aortic Dissection: Results from the GERAADA Registry. J Am Coll Cardiol 65: 2628-2635. Link: http://bit.ly/3t10W94

2. Pacini D, Leone A, Belotti LM, Daniela F, Davide G, et al. (2013) Acute type A aortic dissection: significance of multiorgan malperfusion. Eur J Cardiothorac Surg 43: 820-826. Link: http://bit.ly/3uZxKkO

3. Todd C. Crawford, Robert J. Beaulieu, Bryan A. Ehlert, E. V. Ratchford, James $\mathrm{H}$, et al. (2016) Malperfusion Syndromes in Aortic Dissection. Vasc Med 21: 264-273. Link: http://bit.ly/3sW0Fo3
4. Wasnik A, Kaza RK, Al-Hawary MM, Liu PS, Platt JF (2011) Multidetector CT imaging in mesenteric ischemia-pearls and pitfalls. Emerg Radiol 18: 145156. Link: http://bit.ly/30mk2KM

5. Howard TJ, Plaskon LA, Wiebke EA, Wilcox MG, Madura JA (1996) Nonocclusive mesenteric ischemia remains a diagnostic dilemma. Am J Surg 171: 405-408. Link: http://bit.ly/3rtMtCg

6. Yamashiro S, Kuniyoshi Y, Kise Y, Arakaki R (2012) Delayed viscera malperfusion after Bentall procedure for type $A$ acute aortic dissection. Interact CardioVasc Thorac Surg 15: 794-796. Link: http://bit.ly/2MXxcLa

7. Velayudhan BV, Idhrees AM, Mukesh K, Kannan RN (2019) Mesenteric Malperfusion in Acute Aortic Dissection: Challenges and Frontiers. Semin Thorac Cardiovasc Surg 31: 668-673. Link: http://bit.ly/2O3mZNU

8. Slonim SM, Miller DC, Mitchell RS, CP Semba, MK Razavi, et al. (1999) Percutaneous balloon fenestration and stenting for lifethreatening ischemic complications in patients with acute aortic dissection. J Thorac Cardiovasc Surg 117: 1118-1126. Link: http://bit.ly/3v2UDnu

9. Girardi LN, Krieger KH, Lee LY, Charles AM, Anthony JT, et al. (2004) Management strategies for type A dissection complicated by peripheral vascular malperfusion. Ann Thorac Surg 77: 1309-1314. Link: http://bit. ly/3ele3ho

10. Shiiya N, Matsuzaki K, Kunihara T, Murashita T, Matsui Y (2007) Management of vital organ malperfusion in acute aortic dissection: proposal of a mechanism-specific approach. Gen Thorac Cardiovasc Surg 55: 85-90. Link: http://bit.ly/2MSIgsO

11. Nienaber CA, Fattori R, Lund G, Christoph D, Walter W, et al. (1999) Nonsurgical reconstruction of thoracic aortic dissection by stent-graft placement. N Engl J Med 340: 1539-1545. Link: https://bit.ly/2MUJ3cN

12. Xenos ES, Minion DJ, Davenport DL, Omar H, Nick N Ab, et al. (2009) Endovascular versus open repair for descending thoracic aortic rupture: institutional experience and meta-analysis. Eur J Cardiothorac Surg 35: 282 286. Link: http://bit.ly/3v2Lw6h 